\title{
CURIOSIDADES SOBRE A REAÇÃO ALDÓLICA UTILIZADA COMO ETAPA CHAVE NA SÍNTESE BRASILEIRA DOS ÁCIDOS PTERÍDICOS A E B
}

\author{
Luiz C. Dias* e Airton G. Salles Jr. \\ Instituto de Química, Universidade Estadual de Campinas, CP 6154, 13084-971 Campinas - SP, Brasil
}

Recebido em 23/4/10; aceito em 17/6/10; publicado na web em 18/10/10

\begin{abstract}
CURIOSITIES ABOUT THE ALDOL REACTION EMPLOYED AS A KEY STEP IN THE SYNTHESIS OF PTERIDIC ACIDS A AND B. This work describes an overview of our synthesis of pteridic acids A and B and discloses some interesting results related to the lithium enolate-mediated aldol reaction used as key step to set up the C5-C15 fragment of these natural products. This first example, as far we know, of an aldol reaction between a chiral enolate of a $(Z)$ enone and a chiral aldehyde has driven us to a series of experiments showing the remarkable relation between enolization selectivity and reaction conditions.
\end{abstract}

Keywords: lithium enolates; double stereoinduction; enolization selectivity.

\section{INTRODUÇÃO}

Os ácidos pterídicos A e B (Figura 1) são policetídeos espirocíclicos isolados em pequenas quantidades por Igarashi e colaboradores, a partir da fermentação de uma cultura da bactéria Streptomyces hygroscopicus TP-A0451 obtida das raízes da planta Pteridium aquilinum. ${ }^{1}$

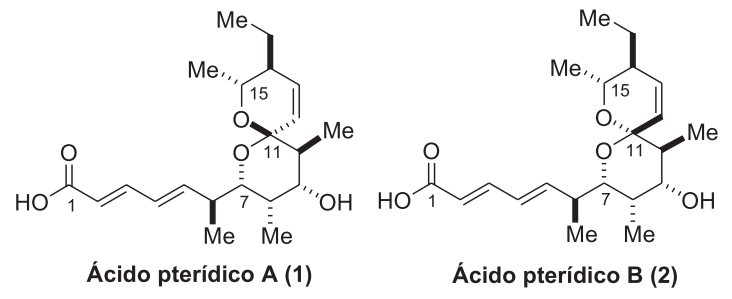

Figura 1. Ácidos pterídicos A e B

A planta Pteridium aquilinum, também conhecida como samambaia-das-taperas, é uma pteridófita que pode atingir até $2 \mathrm{~m}$ de altura, sendo comumente encontrada em solos ácidos. Esta planta apresenta efeitos tóxicos relacionados a problemas neurológicos, cardíacos e hemorrágicos em humanos. Outro efeito tóxico atribuído a esta espécie consiste na carcinogenicidade. Esta última característica foi demonstrada em ratos através da ingestão prolongada de pellets contendo $P$. aquilinum, o que ocasionou múltiplos adenocarcinomas intestinais naqueles animais. ${ }^{2}$ As maiores concentrações de substâncias tóxicas encontram-se nos caules subterrâneos e nos brotos aéreos. ${ }^{3}$

O processo de isolamento dos ácidos pterídicos envolveu sequências de etapas cromatográficas e extrações onde foi possível obter, a partir de $3 \mathrm{~L}$ de extrato bruto, 11,2 mg de ácido pterídico A e 3,5 mg de ácido pterídico B. ${ }^{1} \mathrm{~A}$ atividade biológica atribuída a estes ácidos relaciona-se com a promoção do crescimento de raízes adventíceas em plantas com eficiência comparável à do ácido 3-indolacético (auxina). A concentração efetiva para que estes ácidos exerçam atividade também é comparável à da auxina (da ordem de $1 \mathrm{nM}$ ). Até o momento, foram relatadas na literatura três sínteses totais para os ácidos pterídicos A e B e uma síntese total para o ácido pterídico A.,

*e-mail: 1dias@iqm.unicamp.br

\#Dedicado to Prof. Hans Viertler (IQUSP) por suas contribuições à area de Química no Brasil.
Este trabalho tem como objetivo principal divulgar os interessantes resultados obtidos na reação aldólica intermediada por enolato de lítio empregada na construção do fragmento C5-C15 dos ácidos pterídicos A e B. Um estudo foi realizado demonstrando a íntima relação entre a estereosseletividade na etapa de enolização e variações nas condições experimentais.

\section{RESULTADOS E DISCUSSÃO}

Em 2009, nosso grupo de pesquisas descreveu a síntese total dos ácidos pterídicos A e B através de uma rota sintética curta e eficiente, tendo como etapa-chave uma reação aldólica com a participação de um enolato de lítio. ${ }^{4}$

A estereoquímica relativa syn entre os centros C9 e C10 foi obtida através da reação aldólica otimizada entre a etil cetona $\mathbf{3}$ e o aldeído 4 intermediada por LiHMDS/HMPA e realizada em $70 \%$ de rendimento (Esquema 1). ${ }^{6,7}$ Após purificação por coluna cromatográfica, o aldol $\mathbf{5}$ syn com adição anti-Felkin, inédito na literatura, foi obtido em $56 \%$ de rendimento. A determinação da estereoquímica relativa e absoluta para 5 foi realizada mediante derivatização e, neste caso, este procedimento já é parte da rota sintética, uma vez que os espirocetais $\mathbf{8}$ e $\mathbf{9}$ formados a partir de $\mathbf{5}$ permitiram a obtenção de tais informações (Esquema 2). ${ }^{8-10}$ Outro produto de aldol secundário, tentativamente assinalado como sendo o produto anti $\mathbf{6}$, também foi isolado em $14 \%$ de rendimento (Esquema 1). A estereoquímica relativa para o aldol 6 não havia sido determinada. ${ }^{4}$

Os espirocetais 8 e 9 correspondentes aos ácidos pterídicos A e $\mathrm{B}$, respectivamente, foram obtidos como uma mistura de epímeros, separável por cromatografia flash, na proporção de 50:50 (determinada pela análise de $\mathrm{RMN} \mathrm{de}{ }^{13} \mathrm{C}$ do produto bruto) (Esquema 2). ${ }^{11,12}$

A síntese total dos ácidos pterídicos A e B foi completada e a rota sintética planejada conduziu ao ácido pterídico A em 2,9\% de rendimento global para 13 etapas e ao ácido pterídico B em 2,8\% de rendimento global também para 13 etapas (Esquema 2). ${ }^{4}$ Esta rota sintética chama a atenção pela nova e interessante abordagem na obtenção do fragmento C5-C15 via reação aldólica intermediada por lítio. Até onde sabemos este é o primeiro exemplo da utilização de um enolato de uma etil cetona $\alpha, \beta$-insaturada quiral com geometria $Z$ em uma reação aldólica. 


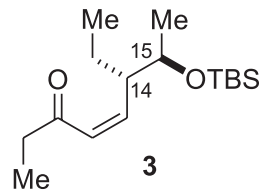

$$
\begin{aligned}
& \text { LiHMDS, THF } \\
& \text { HMPA, }-78^{\circ} \mathrm{C} \\
& \text { PMBO } 7=\mathrm{H} \\
& 4 \mathrm{Me} \mathrm{Me} \\
& -78^{\circ} \mathrm{C}, 70 \%
\end{aligned}
$$<smiles>CCCCCC(C)OCC(C)C</smiles>

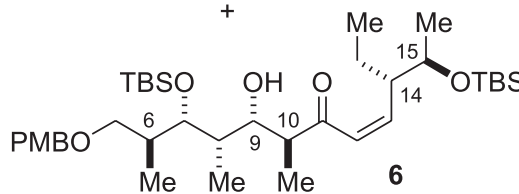

ds (syn, adição anti-Felkin 5:anti, adição Felkin 6) = 80:20

Esquema 1. Obtenção do fragmento C5-C15 (5) via reação aldólica intermediada por enolato de lítio

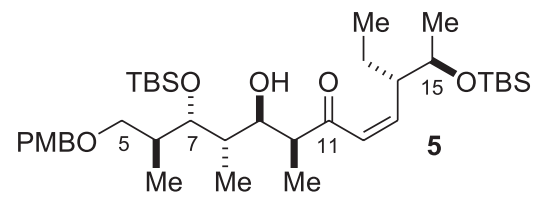

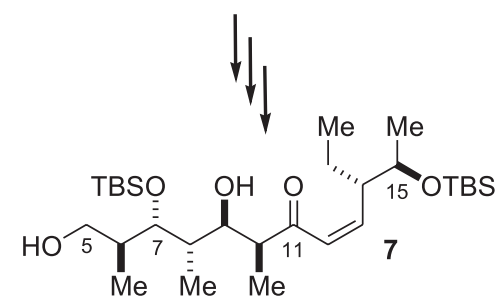

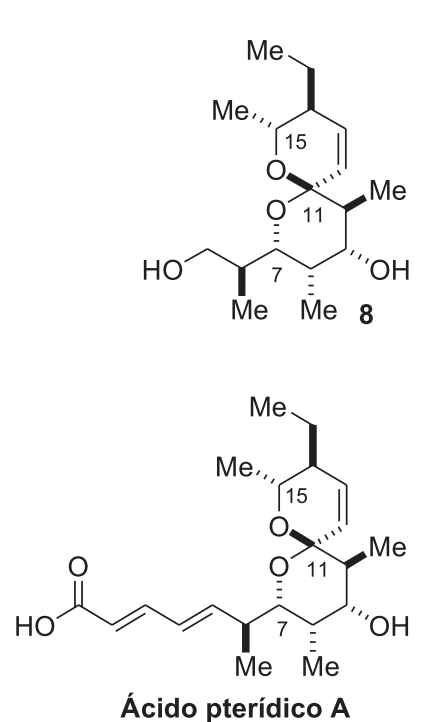

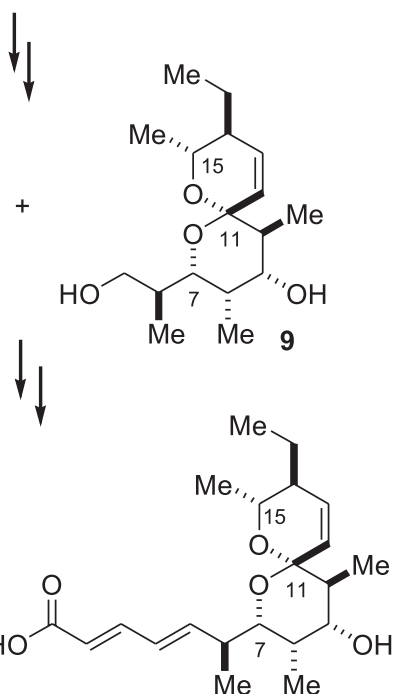

Ácido pterídico A

\section{Ácido pterídico B}

Esquema 2. Síntese dos ácidos pterídicos $A$ e $B$

\section{Confirmação da estereoquímica relativa do aduto de aldol secundário 6 via derivatização}

Diante da incerteza quanto à estereoquímica relativa do produto secundário 6 (Esquema 1), obtido na reação aldólica intermediada

por lítio, procedemos à derivatização do mesmo através da ciclização para o correspondente espirocetal 10, sendo que estes resultados não haviam sido descritos anteriormente (Esquema 3). A desproteção dos éteres de silício do aldol 6 utilizando-se HF-piridina em THF conduziu a apenas um produto de ciclização, o espirocetal 10, também inédito na literatura, em $65 \%$ de rendimento.

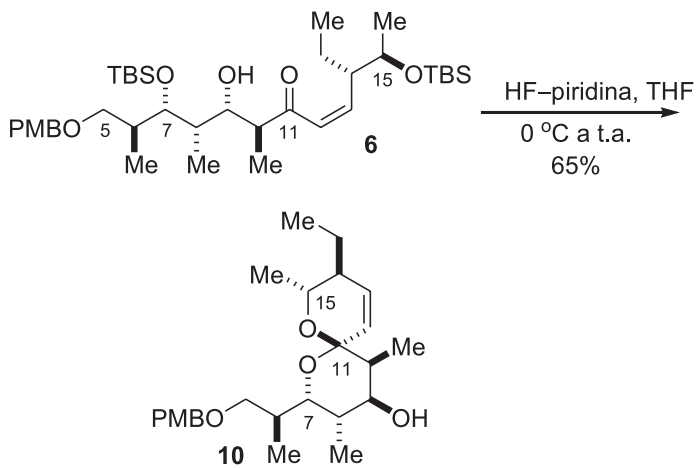

Esquema 3. Obtenção do espirocetal 10 via ciclização do aldol secundário 6

A análise dos dados de $\mathrm{RMN}$ de ${ }^{1} \mathrm{H}$ do espirocetal 10 confirmou a existência do aldol anti com adição de Felkin $\mathbf{6}$ como produto secundário na reação aldólica intermediada por lítio (Tabela 1).

Tabela 1. Dados de RMN de ${ }^{1} \mathrm{H}$ para os hidrogênios H9, H10 e H15 em 10

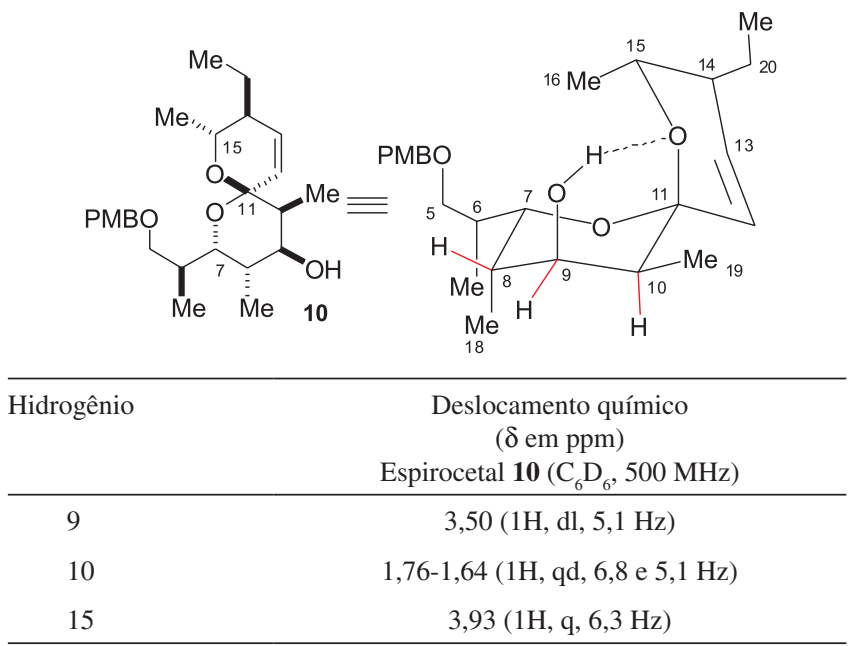

A análise dos dados de $\mathrm{RMN}$ de ${ }^{13} \mathrm{C}$ mostra a presença de um sinal típico de espirocetal com duplo efeito anomérico, visto que o deslocamento químico para o C11 é $97,4 \mathrm{ppm} .{ }^{12}$ Este valor se aproxima bastante do valor observado para o espirocetal 8 (97,2 ppm), o qual também apresenta duplo efeito anomérico. Um fato interessante foi a obtenção de apenas um espirocetal como produto. Uma análise espacial mais detalhada revelou a possibilidade de ligação de hidrogênio intramolecular entre a hidroxila em C9 e o oxigênio do anel insaturado, formando um anel de seis membros (Tabela 1). Esta interação, existente apenas na conformação indicada e possível apenas no espirocetal proveniente do aldol anti com adição Felkin, responderia pela formação de um único espirocetal favorecido termodinamicamente.

\section{Estereoindução simples envolvendo a etil cetona 3}

Apesar dos elementos de estereoindução mencionados até o momento envolverem apenas os centros estereogênicos do alde- 
ído e a geometria do enolato da etil cetona $\mathbf{3}$, pode-se imaginar a possibilidade de um determinado controle do caminho estereosseletivo por parte dos centros estereogênicos da etil cetona. ${ }^{9}$ Estes centros estariam um tanto distantes do centro reacional, mas diante da comprovação da existência de induções remotas e mesmo ultrarremotas esta possibilidade adquire um realismo a se considerar. ${ }^{13,14}$ Além disto, a etil cetona 3 não havia sido, até a publicação de nosso trabalho, descrita na literatura. Assim, acreditamos que ainda existam características muito particulares a esta a serem determinadas, as quais podem esclarecer alguns resultados obtidos.

Inicialmente, foi investigada a causa da obtenção de um aldol anti como produto secundário na reação intermediada por lítio. Estaria esta relacionada à equilibração entre os produtos de aldol? Ou estaria relacionada à seletividade na etapa de enolização da etil cetona 3? Para tanto, procedemos à derivatização da etil cetona 3 para o correspondente silil enol éter. Visando a determinação da seletividade $E / Z$ inerente à etil cetona 3 , realizamos a enolização sem o emprego de HMPA e em concentração diferente da utilizada na reação aldólica. O objetivo foi, além de determinar a geometria do enolato formado, mostrar como a variação das condições de enolização pode afetar intensamente a seletividade $E / Z$ do processo.

A enolização da etil cetona 3 utilizando-se LiHMDS (1,0 mol $\left.\mathrm{L}^{-1}\right)$ seguido de reação com TMSCl nas condições descritas por Xie e colaboradores (Esquema 4, condição reacional I, Tabela 2) forneceu uma mistura de silil enol éteres 11, que foi analisada por RMN de ${ }^{1} \mathrm{H}$ sem prévia purificação. ${ }^{15}$

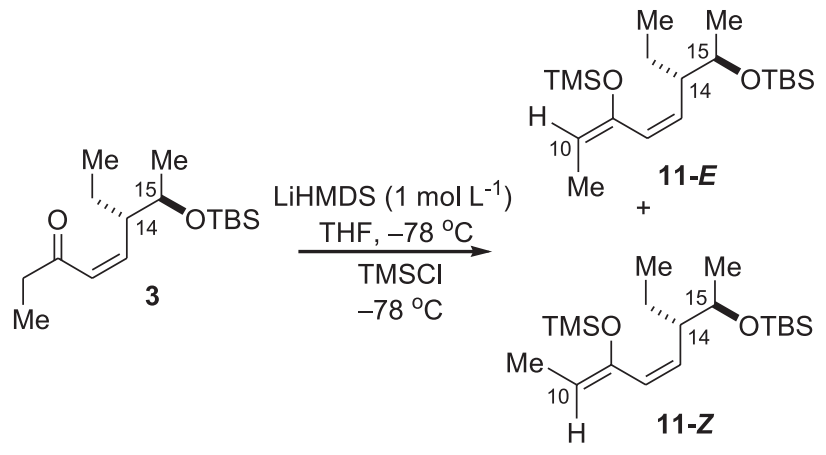

Esquema 4. Obtenção do silil enol éter 11. Condição reacional I
Tabela 2. Condição reacional I utilizada para enolização da etil cetona 3

\begin{tabular}{ll}
\hline Condição reacional I & \\
\hline Cetona & 1 equiv. \\
LiHMDS & 1,1 equiv. \\
Concentração da mistura reacional & $0,16 \mathrm{~mol} \mathrm{~L}^{-1}$ \\
TMSCl & 1,2 equiv. \\
HMPA & - \\
\hline
\end{tabular}

Os dados de RMN de ${ }^{1} \mathrm{H}$ para $\mathbf{1 1}$ mostraram a presença de dois quartetos em 4,79 e 4,99 ppm (Figura 2). Estes sinais seriam referentes aos hidrogênios vinílicos H10 nos silil enol éteres de ambas as geometrias. A relação, obtida por integração dos picos, entre o sinal mais protegido e o mais desprotegido é de 75:25, respectivamente (Figura 2). Apenas considerando este resultado é possível perceber que o processo de enolização desta etil cetona em particular não é altamente seletivo, mesmo considerando a utilização da base LiHMDS o qual, notoriamente, permite enolizações $Z$-seletivas. A estrutura desta etil cetona parece interferir ativamente na seletividade da enolização. É importante salientar que esta condição de enolização não é a mesma utilizada na reação aldólica para síntese dos ácidos pterídicos.

Entre os métodos utilizados para a determinação da geometria de silil enol éteres, merecem destaque o NOE diferencial e a análise do espectro de RMN de ${ }^{13} \mathrm{C} .{ }^{16-18}$ No entanto, diante da separação clara entre os sinais dos hidrogênios vinílicos $\mathrm{H} 10$ em ambos os silil enol éteres, optamos por investigar a reação, a princípio, utilizando apenas a análise de $\mathrm{RMN}$ de ${ }^{1} \mathrm{H}$. Aplicando o método descrito por House e colaboradores para tal análise pudemos obter informações interessantes ${ }^{19}$ Os autores investigaram vários outros silil enol éteres e apontaram que o hidrogênio vinílico em um diastereoisômero $Z$, geralmente, aparece em menor deslocamento químico (mais protegido) que o correspondente em um diastereoisômero $E .{ }^{19}$ Desta forma, o sinal em 4,79 ppm seria referente ao hidrogênio vinílico H10 do silil enol éter 11-Z. O sinal em 4,99 ppm, por sua vez, corresponderia ao hidrogênio vinílico H10 do silil enol éter 11-E (Figura 2).

Aparentemente, a enolização foi moderadamente seletiva, favorecendo o silil enol éter de geometria $Z$ em razão de 75:25. Considerando que o emprego de HMPA como cossolvente pode tornar a enolização mais seletiva favorecendo o silil enol éter de geometria

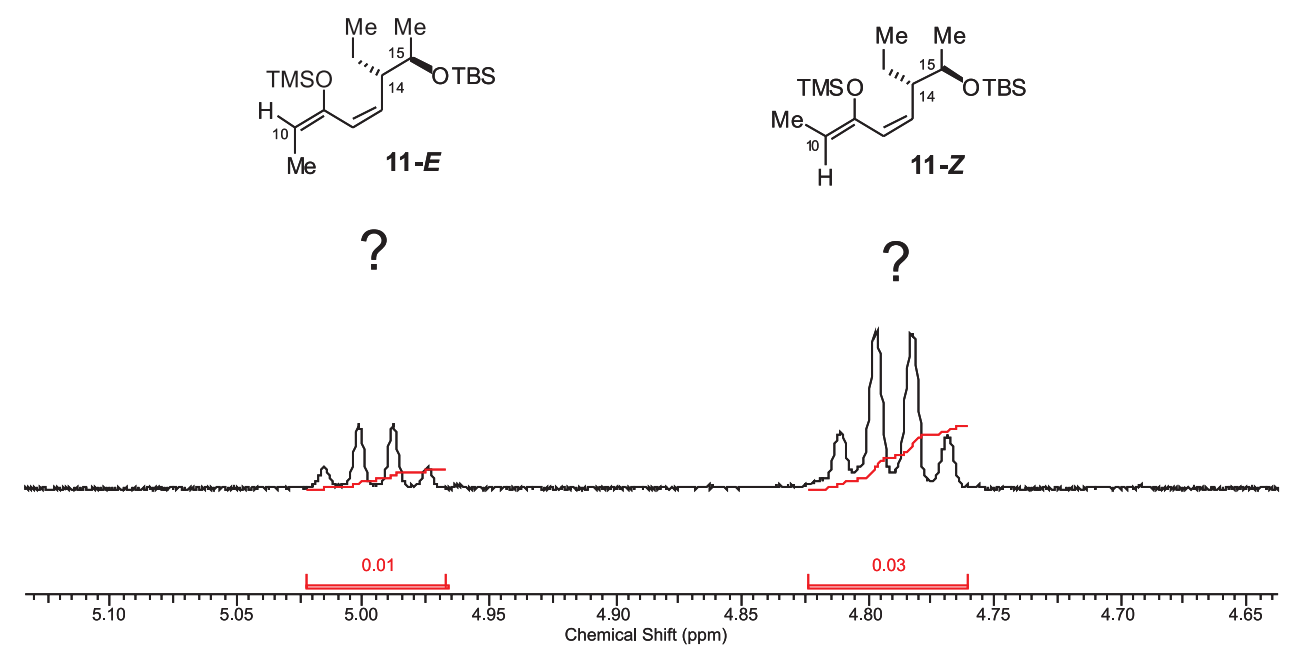

Figura 2. Parte do espectro de $R M N$ de ${ }^{l} \mathrm{H}\left(500 \mathrm{MHz}, \mathrm{C}_{6} \mathrm{D}_{6}\right)$ para 11 obtido na condição reacional I. Sinais referentes aos hidrogênios vinílicos $\mathrm{H} 10$ nos silil enol éteres de ambas as geometrias 


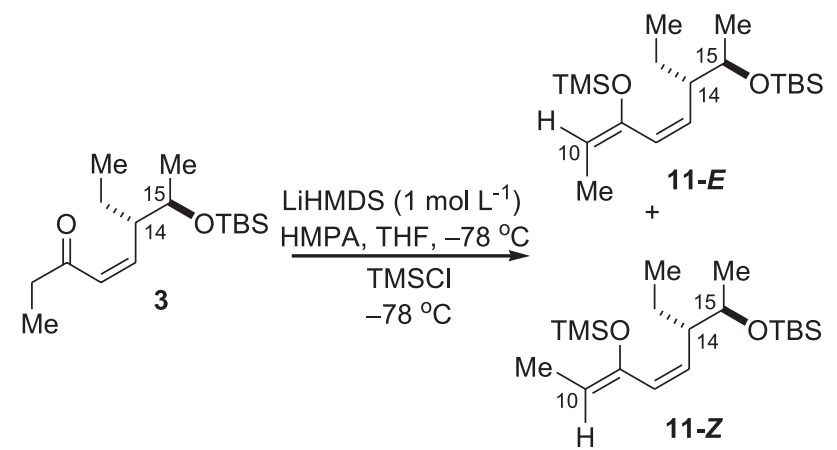

Esquema 5. Obtenção do silil enol éter 11. Condição reacional II

Tabela 3. Condição reacional II utilizada para enolização da etil cetona 3

\begin{tabular}{lc}
\hline Condição reacional II & \\
\hline Cetona & 1,0 equiv. \\
LiHMDS & 1,1 equiv. \\
Concentração da mistura reacional & 0,16 mol L $^{-1}$ \\
TMSCl & 1,2 equiv. \\
HMPA & 3,0 equiv. \\
\hline
\end{tabular}

$Z$, repetimos a reação nas mesmas condições apontadas, porém, na presença de HMPA (Esquema 5, condição reacional II, Tabela 3).

Surpreendentemente, a análise dos dados de RMN de ${ }^{1} \mathrm{H}$ mostrou um resultado interessantíssimo. O quarteto em 4,99 ppm aumentou sua área de integração em relação ao quarteto em 4,79 ppm (Figura $3)$. No entanto, este sinal mais desprotegido deveria ser atribuído ao hidrogênio vinílico $\mathrm{H} 10$ para o silil enol éter de geometria $E$, segundo a regra de House. Sabendo que o emprego de HMPA favorece enolizações $Z$-seletivas, este fato nos levou a imaginar que o sinal mais desprotegido $(4,99 \mathrm{ppm})$ deveria ser do silil enol éter de geometria $Z$ e não de geometria $E$, violando, assim, o padrão determinado por House e colaboradores. A relação, obtida por integração dos picos, entre o sinal mais protegido e o mais desprotegido agora é de aproximadamente 50:50 mostrando uma enolização não-seletiva (Figura 3).

A perda de seletividade, neste caso, pode ter sido ocasionada pelo favorecimento da enolização Z-seletiva em HMPA. Seguindo uma linha lógica, repetimos a reação nas mesmas condições de enolização da reação aldólica utilizada na síntese dos ácidos pterídicos (Esquema 6, condição reacional III, Tabela 4).

Nesta situação, a concentração da solução foi alterada drasticamente, sendo reduzida de 0,20 para $0,03 \mathrm{~mol} \mathrm{~L}^{-1}$. A análise dos dados

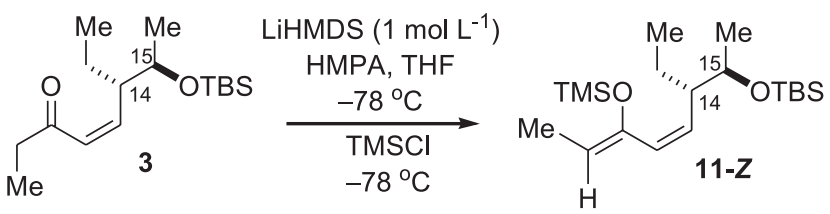

Esquema 6. Obtenção do silil enol éter 11-Z. Condição reacional III

Tabela 4. Condição reacional III utilizada para enolização da etil cetona 3

\begin{tabular}{lc}
\hline Condição reacional III & \\
\hline Cetona & 1,0 equiv. \\
LiHMDS & 1,2 equiv. \\
Concentração da mistura reacional & $0,030 \mathrm{~mol} \mathrm{~L}^{-1}$ \\
TMSCl & 1,2 equiv. \\
HMPA & 3,0 equiv. \\
\hline
\end{tabular}

de RMN de ${ }^{1} \mathrm{H}$ mostrou que o processo de enolização foi altamente seletivo. Apenas o quarteto em 4,99 ppm surgiu no espectro de RMN de ${ }^{1} \mathrm{H}$ (Figura 4).

Esta pequena sequência de experimentos mostrou como a reação de enolização intermediada por lítio é extremamente sensível às variações das condições reacionais. Aparentemente, na condição reacional III, pudemos obter o silil enol éter de geometria $Z$ seletivamente, o que explica a formação preferencial do aldol syn na reação aldólica utilizada na síntese dos ácidos pterídicos. Daí, conclui-se que o aldol anti obtido na mesma reação só pode ser proveniente de uma competição entre os estados de transição competitivos syn e anti.

Naturalmente, a confirmação da geometria do silil enol éter deve ser feita por métodos mais confiáveis. Para tanto, obtivemos espectros de NOE diferencial (Material suplementar) para o silil enol éter 11-Z obtido na condição reacional III (Figura 4). Neste experimento, a irradiação do hidrogênio vinílico H10 ( $\delta$ 4,99 ppm) conduziu a um incremento de $4,8 \%$ nos hidrogênios da metila vinílica $(\delta 1,69 \mathrm{ppm})$ e nenhum incremento associado aos hidrogênios das metilas ligadas ao silício $(\delta 0,21 \mathrm{ppm})$. A irradiação dos hidrogênios da metila vinílica $(\delta 1,69 \mathrm{ppm})$ também conduziu a incrementos de 4,9 e $4,7 \%$ nos sinais dos hidrogênios das metilas do grupo TMS ( $\delta 0,21 \mathrm{ppm})$ e no sinal do hidrogênio vinílico H10 ( $\delta$ 4,99 ppm), respectivamente (Figura 4).

Este resultado está em acordo com o previsto nos estudos de Keller e colaboradores ${ }^{16}$ sobre a determinação da geometria de silil enol éteres por NOE diferencial. Segundo estes autores, a irradiação do hidrogênio vinílico no diastereoisômero $Z$ ocasiona um incremento

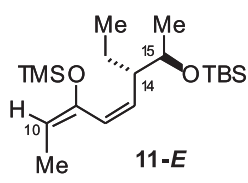

!!!
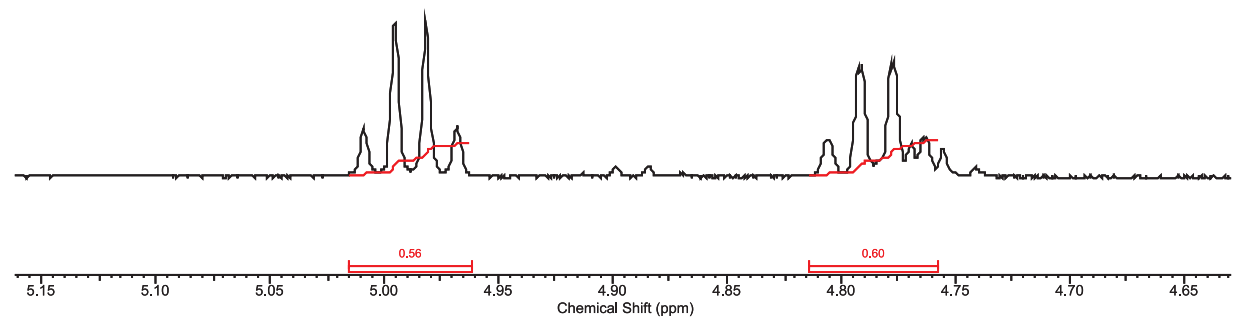

Figura 3. Parte do espectro de $R M N$ de ${ }^{l} \mathrm{H}$ para a mistura de 11 (500 MHz, $\left.C_{6} D_{6}\right)$ obtido na condição reacional II 


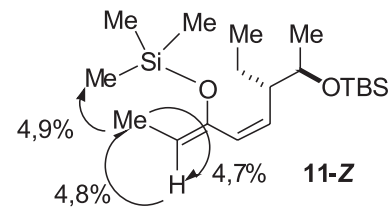

!!!

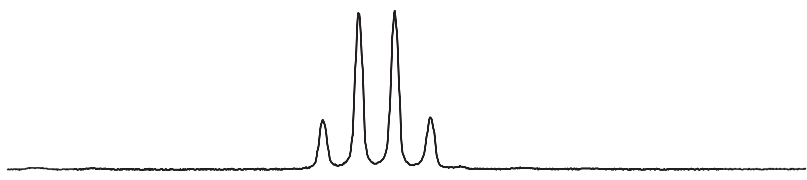

$\begin{array}{cccccccc}5.10 & 5.05 & 5.00 & 4.95 & 4.90 & 4.85 \\ & & \text { Chemical Shift (ppm) }\end{array}$

Figura 4. Parte do espectro de $R M N$ de ${ }^{1} \mathrm{H}$ para $\mathbf{1 1 - Z}\left(500 \mathrm{MHz}, \mathrm{C}_{6} \mathrm{D}_{6}\right)$ e incrementos resultantes dos espectros de NOE diferencial para o silil enol éter obtido na condição reacional III

apenas no sinal da metila vinílica, enquanto que incrementos nesta mesma metila e nos hidrogênios da metila do grupo TMS são observados no diastereoisômero $E$. De maneira correspondente, a irradiação dos hidrogênios da metila vinílica ocasiona um incremento no sinal do hidrogênio vinílico e dos hidrogênios do grupo TMS.

Desta forma, percebe-se que, neste tipo de sistema, as generalizações feitas por House e colaboradores não se aplicam. ${ }^{19}$ Provavelmente, silil enol éteres como estes, os quais apresentam uma porção dieno conjugado, possuam características muito peculiares e diferentes daqueles estudados por House (Material suplementar, Figura 13S).

Ainda tentando determinar a possível causa da obtenção do aldol anti nas condições reacionais utilizadas para a reação aldólica, realizamos uma pequena investigação visando observar alguma indução associada à etil cetona 3 . Desta forma, a reação entre isobutiraldeído e o enolato de lítio da etil cetona 3 foi executada (Esquema 7). A con-<smiles>C=C(C(=O)CC)[C@@H](CC)[C@H](C)[OH+]CC</smiles>

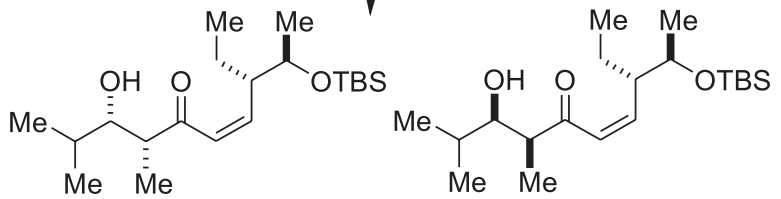<smiles>CC[C@H](/C=C\C(=O)[C@@H](C)[C@H](O)C(C)C)[C@@H](C)O[SbH3]</smiles><smiles>CC[C@H](/C=C\C(=O)[C@@H](C)[C@H](O)C(C)C)[C@@H](C)O[SbH3]</smiles>

Esquema 7. Reação aldólica entre isobutiraldeído e a etil cetona 3 intermediada por enolato de lítio dição reacional III, seletiva para o enolato $Z$, foi utilizada neste caso.

Foram obtidos quatro possíveis adutos aldol em rendimento de $80 \%$ para a mistura de isômeros, sendo recuperado o material de partida. As manchas referentes aos isômeros possuíam $R_{f}$ muito próximos em CCD e foram isoladas em conjunto. A análise dos dados de $\mathrm{RMN}$ de ${ }^{1} \mathrm{H}$ da mistura de isômeros mostrou a aparente presença de apenas dois isômeros, como pode ser observado pelos sinais dos hidrogênios $\alpha$-carbonílicos (Material suplementar, Figura 14S). No entanto, os dados de $\mathrm{RMN}$ de ${ }^{13} \mathrm{C}$ mostraram a presença de quatro sinais referentes à carbonila, indicando a existência dos quatro possíveis diastereoisômeros.

Estes são dois adutos de aldol com estereoquímica relativa syn e dois adutos de aldol com estereoquímica relativa anti, mostrando que não houve indução por parte do enolato de lítio da etil cetona 3. A diferença de proporção (2:1) entre os sinais no espectro de RMN de ${ }^{1} \mathrm{H} \mathrm{e}^{13} \mathrm{C}$ (Material suplementar, Figura 14S) pode se dever à presença de uma maior quantidade de aldol syn, uma vez que a enolização é $Z$-seletiva. $\mathrm{O}$ sinal em menor proporção é referente ao aldol anti obtido através de um estado de transição anti competitivo com o estado de transição syn, que leva ao isômero obtido em maior proporção, visto que a condição reacional utilizada permite este processo. Estes resultados mostram que não há preferência facial significativa por parte do enolato e o aldeído deve desempenhar um papel extremamente importante neste processo.

\section{CONCLUSÃO GERAL}

Este trabalho se apoiou, essencialmente, sobre um tipo de química extremamente interessante, porém um pouco esquecida, sob nosso ponto de vista, nos últimos anos: a química dos enolatos de lítio. Estas espécies despontaram nos anos 70 como intermediários úteis em síntese orgânica, principalmente em reações de alquilação. As primeiras reações aldólicas com utilidade sintética continham, em seu cerne, enolatos de lítio. No entanto, reações envolvendo estas espécies foram cedendo espaço para outras metodologias, porém sem esgotar todo o seu potencial. Provavelmente, um grande empecilho que pode se transformar, no futuro, em uma fonte de variabilidade metodológica é a considerável influência da molaridade e do tipo de solvente utilizado nos resultados. Sem um estudo sistemático para este tema, a tendência é o abandono do método, uma vez que a sintonia fina das condições reacionais se torna difícil assim como a previsão dos produtos obtidos. Os níveis de agregação nos enolatos de lítio também merecem destaque. O controle sobre esta característica supramolecular é sem dúvida um desafio, mas pode trazer, como frutos, variações na estereosseletividade dependentes apenas do estado de agregação. A viagem pelo universo dos enolatos de lítio não pode ser depreciada e pede por mais participação nos planejamentos sintéticos.

\section{MATERIAL SUPLEMENTAR}

Procedimentos experimentais, figuras e espectros completos encontram-se disponíveis gratuitamente em http://quimicanova.sbq. org.br, na forma de arquivo PDF.

\section{REFERÊNCIAS}

1. Ygarashi, Y.; Yoshida, R.; Furumai, T.; J. Antibiot. 2002, 55, 764. A numeração para os carbonos dos ácidos pterídicos segue a mesma adotada nesta referência.

2. Evans, I. A.; Mason, J.; Nature 1965, 208, 913.

3. Centro de Informação Toxicológica do Rio Grande do Sul, http://www. cit.rs.gov.br, acessada em Março 2010. 
4. Dias, L. C.; Salles Jr., A. G.; J. Org. Chem. 2009, 74, 5584; Rocha, D. R.; Rev. Virtual Quim. 2009, 1, 182; Nakahata, T.; Fujimura, S.; Kuwahara, S.; Chem. Eur. J. 2006, 12, 4584; Paterson, I.; Anderson, E. A.; Findlay, A. D.; Knappy, C. S.; Tetrahedron 2008, 64, 4768; Nakahata, T.; Kuwahara, S.; Chem. Commun. 2005, 1028.

5. Yadav, J. S.; Rajender, V.; Gangadhara Rao, Y.; Org. Lett. 2009, 12, 348.

6. Evans, D. A.; Bartroli, J.; Shih, T. L.; J. Am. Chem. Soc. 1981, 103, 2127; Evans, D. A.; Taber, T. R.; Tetrahedron Lett. 1980, 21, 4675.

7. Dias, L. C.; Lima, D. J. P.; Gonçalves, C. C. S.; Andricopulo, A. D.; Eur. J. Org. Chem. 2009, 1491; Smith III, A. B.; Beauchamp, T. J.; LaMarch, M. J.; Kaufman, M. D.; Qiu, Y.; Arimoto, H.; Jones, D. R.; Kobayashi, K.; J. Am. Chem. Soc. 2000, 122, 8654; Dias, L. C.; de Sousa, M. A.; Tetrahedron Lett. 2003, 44, 5625.

8. Dias, L. C.; de Oliveira, L. G.; de Sousa, M. A.; Org. Lett. 2003, 5, 265; Dias, L. C.; Meira, P. R. R.; Tetrahedron Lett. 2002, 43, 185; Dias, L. C.; Meira, P. R. R.; J. Org. Chem. 2005, 70, 4762; Dias, L. C.; de Oliveira, L. G.; Vilcachagua, J. D.; Nigsch, F.; J. Org. Chem. 2005, 70, 2225; Dias, L. C.; de Oliveira, L. G.; Org. Lett. 2001, 3, 3951; Dias, L. C.; Giacomini, R.; Tetrahedron Lett. 1998, 39, 5343; Dias, L. C.; Ferreira, A. A.; Diaz, G.; Ferreira, E.; Meira, P. R. R.; Synthesis 2003, 603.

9. Seebach, D.; Chow, H. F.; Jackson, R. F. W.; Sutter, M. A.; Thaisrivongs, S.; Zimmerman, J.; Liebigs Ann. Chem. 1986, 1281; Frater, G.; Müller, U.; Günther, W.; Tetrahedron 1984, 40, 1269; Evans, D. A.; Fitch, D. M.; J. Org. Chem. 1997, 62, 454.

10. Gustin, D. J.; VanNieuwenhze, M. S.; Roush, W. R.; Tetrahedron Lett. 1995, 36, 3443; Roush, W. R.; J. Org. Chem. 1991, 56, 4151; Evans, D. A.; Dart, M. J.; Duffy, J. L.; Rieger, D. L.; J. Am. Chem. Soc. 1995, 117, 9073; Wu, B.; Liu, Q.; Jin, B.; Qu, T.; Sulikowski, G. A.; Eur. J. Org. Chem. 2006, 277; Lister, T.; Perkins, M. V.; Org. Lett. 2006, 8, 1827; Gillingham, D. G; Hoveyda, A. H.; Angew. Chem., Int. Ed. 2007, 46, 3860; Evans, D. A.; Yang, M. G.; Dart, M. J.; Duffy, J. L.; Tetrahedron Lett. 1996, 37, 1957; Ghidu, V. P.; Wang, J; Wu, B.; Liu, Q.; Jacobs, A.; Marnett, L. J.; Sulikowiski, G. A.; J. Org. Chem. 2008, 73, 4949.
11. Revisões sobre espirocetais: Aho, J. E.; Pihko, P. M.; Rissa, T. K.; Chem. Rev. 2005, 105, 4406; Perron, F.; Albizati, K. F.; Chem. Rev. 1989, 89, 1617; Vaillancourt, V.; Pratt, N. E.; Perron, F; Albizati, K. F. Em The Total Synthesis of Natural Products; Apsimon, J., ed.; John Wiley \& Sons: New York, 1992, vol. 8, p. 533; Mead, K. T.; Brewer, B. N.; Curr. Org. Chem. 2002, 6, 1; Espirocetais em insetos: Francke, W.; Kitching, W.; Curr. Org. Chem. 2001, 5, 233; Bis-espirocetais: Brimble, M. A.; Molecules 2004, 9 , 394; Brimble, M. A.; Furkert, D. P.; Curr. Org. Chem. 2003, 7, 1; Francke, W.; Schröder, W.; Curr. Org. Chem. 1999, 3, 407.

12. Dias, L. C.; de Oliveira, L. G.; Org. Lett. 2004, 6, 2587; de Oliveira, L. G.; Dias, L. C.; Sakauchi, H.; Kiyota, H.; Tetrahedron Lett. 2006, 47, 2413; Dias, L. C.; Correia, V. G.; Finelli, F. G.; Tetrahedron Lett. 2007, 48, 7683; Paterson, I.; Florence, G. J.; Gerlach, K.; Scott, J. P.; Sereinig, N.; J. Am. Chem. Soc. 2001, 123, 9535.

13. Indução 1,5-anti: Dias, L. C.; Pinheiro, S. M.; de Oliveira, V. M.; Ferreira, M. A. B.; Tormena, C. F.; Aguilar, A. M.; Zuckerman-Schpector, J.; Tiekink, E. R. T.; Tetrahedron 2009, 65, 8714; Dias, L. C.; Aguilar, A. M.; Chem. Soc. Rev. 2008, 37, 451; Dias, L. C.; Aguilar, A. M.; Quim. Nova 2007, 30, 2007; Cowden, C. J.; Paterson, I.; Org. React. 1997, 51, 1; Franklin, A. S.; Paterson, I.; Contemp. Org. Synth. 1994, 1, 317.

14. Clayden, J.; Lund, A.; Vallverdú, L.; Helliwell, M.; Nature 2004, 431, 966.

15. Xie, L.; Isenberger, K. M.; Held, G.; Dahl, L. M.; J. Org. Chem. 1997, 62,7516 .

16. Keller, T. H.; Neeland, E. G.; Weiler, L.; J. Org. Chem. 1987, 52, 1870.

17. Heathcock, C. H.; Buse, C. T.; Kleschick, W. A.; Pirrung, M. C.; Sohn, J. E.; Lampe, J.; J. Org. Chem. 1980, 45, 1066.

18. Heathcock, C. H.; Pirrung, M. C.; Sohn, J. E.; J. Org. Chem. 1979, 44, 4294; Evans, D. A.; Nelson, J. V.; Taber, T.; Top. Stereochem. 1982, 13, 1; Dias, L. C.; Aguilar, A. M.; Salles Jr., A. G.; Steil, L. J.; Roush, W. R.; J. Org. Chem. 2005, 70, 10461; Dias, L. C.; Meira, P. R. R.; Ferreira, E.; Org. Lett. 1999, 1, 1335.

19. House, H. O.; Czuba, L. J.; Gall, M.; Olmstead, H. D.; J. Org. Chem. 1969, 34, 2324. 